\title{
Aplicación de la teoría de carteras con activos numismáticos y metales preciosos
}

\section{Portfolio theory application wtih gold numismatic assets and precious metal}

\author{
Camilo Prado Román ${ }^{1}$ \\ José Luis Coca Pérez ${ }^{2}$ \\ Pablo García Estévez ${ }^{1}$ \\ Universidad Rey Juan Carlos (España) / Universidad de Extremadura (España)
}

Recibido el 18 de enero de 2010 y aceptado el 1 de junio de 2010

$\mathrm{N}^{\circ}$ de clasificación JEL: G11

DOI: $10.5295 / \mathrm{cdg} .100201 \mathrm{cp}$

\section{Resumen:}

El objetivo del trabajo es la construcción de diferentes carteras compuestas por activos numismáticos de oro y metales nobles (oro, plata, platino, paladio y rodio); con el fin de construir aquella cartera que mejor se adapte al inversor, acorde a su perfil y conocer cuál es la Cartera del Mercado. Para ello, mediante la Teoría de Carteras (Markowitz, 1952; 1959), construiremos la Frontera Eficiente y trazaremos la Línea del Mercado de Capitales o CML. El programa utilizado es la Toolkit financiera del MatLab. La muestra utilizada son los activos numismáticos de oro emitidos por España, EEUU, Gran Bretaña y Francia desde 1900 a 2009 y el periodo de estudio es del 2003 al 2009. Los resultados obtenidos nos confirman, aquellos activos no financieros seleccionados para construir la cartera eficiente y conocer la cartera del Mercado.

Palabras clave:

Bien de colección, metales nobles, Teoría de Carteras, Cartera del Mercado.

\section{Abstract:}

Our objective is to build different Precious Metals (gold, silver, palladium, platinum and rhodium) and Gold Numismatic Asset Portfolios. The purpose is being able to build the best portfolio for the different investors and to know The Market Portfolio. For that, by means of The Portfolio Theory methodology (Markowitz, 1952; 1959), we build the efficient frontier and we will trace the Capital Market Line, CML. The program we use is Matlab (financial Toolkit). Research sample is composed by gold numismatic assets and precious metals. Those assets have been issued by Spain, USA, Great Britain and France from 1900 to 2009, and the research period is to 2003-2009. The results obtained confirm those not financial assets selected to build the efficient portfolio and the Market Portfolio.

\footnotetext{
${ }^{1}$ Universidad Rey Juan Carlos. Facultad de Ciencias Jurídicas y Sociales, Paseo de los artilleros, s/n, 28032, Madrid. Email: camilo.prado.roman@urjc.es y pablo.garcia.estevez@urjc.es

${ }^{2}$ Universidad de Extremadura, Facultad de Ciencias Empresariales y Turismo, Av. Universidad, s/n, 10071, Cáceres. Email: jlcocap@hotmail.com
} 


\section{Keywords:}

Collectible Tangible Asset, Precious Metals, Portfolio Theory, Market Portfolio. 


\section{INTRODUCCIÓN}

Las inversiones alternativas son aquellas orientadas a obtener una rentabilidad absoluta, positiva e independiente de la evolución de los mercados. López y Hurtado (2008), la definen como "aquella orientada a obtener retornos positivos en cualquier entorno de mercado. Estos retornos suelen tener una baja correlación con el comportamiento de los activos financieros tradicionales". Dentro de las inversiones alternativas se incluyen el capital riesgo, los Hedge Funds y los activos no financieros. Las Inversiones en activos no financieros, están compuestas por todos aquellos activos cuyo rendimiento no está directamente relacionado con la evolución de los mercados financieros, entendiendo por mercado financiero (mercados monetarios, renta fija y renta variable). Estas inversiones tendrán: Una gestión no tradicional, una baja correlación con la gestión tradicional y altos Ratios Sharpe (Bolsa y Mercados Españoles et al, 2007). Como función principal, intentan reducir la correlación entre los distintos mercados financieros. Si los introducimos en una cartera de inversión, tendrán las funciones de diversificación del riesgo y la de obtención de retornos en términos absolutos. Los activos no financieros están formados por los activos de colección, los inmuebles, las materias primas, los metales nobles y otros como los recursos naturales.

Los activos de colección o bienes de colección son activos no financieros que se engloban dentro de las inversiones no financieras. Dentro de ellos, deben identificarse aquellos bienes que por sus características específicas que los distinguen de los demás, tales como, su carácter único, su escasez, su rareza, su estimación singular (especificidad, antigüedad, merito artístico, etc.) y/o su estimación global, provoquen que vayan paulatinamente aumentando su valor a lo largo del tiempo.

Los activos numismáticos, activos no financieros, están relacionados con la inversión en monedas, en sus tres tipos, históricas, conmemorativas y bullions. Los activos numismáticos de oro, activos utilizados en el estudio, están compuestos por las monedas históricas de oro, aquellas que han estado o están en circulación; las monedas conmemorativas de oro, aquellas emitidas por un gobierno para conmemorar algún suceso; y las monedas bullion de oro, aquellas monedas emitidas en metales preciosos, en nuestro caso el oro, cuyo precio lo marca el metal de acuñación mas una prima de entre 3\%-6\%. Los metales nobles utilizados en el estudio (oro, plata, platino, paladio y rodio) son un grupo de metales muy inertes químicamente, es decir, que no reaccionan químicamente o que reaccionan muy poco con otros compuestos químicos, lo que les da una gran utilidad para muchos fines tecnológicos, joyería, y por ende, para la inversión (Jeffrey, 2000).

El objetivo del trabajo es la construcción de diferentes carteras de inversión compuestas por activos numismáticos de oro, y los metales nobles (oro, plata, platino, paladio y rodio), así podremos conocer que activos son utilizados para la construcción de carteras eficientes, con el fin de proporcionarle al inversor aquella cartera que mejor se adapte al a su perfil; además de conocer la Cartera del Mercado. Para ello, mediante el uso de la Teoría de Carteras (Markowitz, 1952; 1959), construiremos la Frontera Eficiente y trazaremos la Línea del Mercado de Capitales. 


\section{INVERSIÓN EN BIENES DE COLECCIÓN}

La inversión en activos no financieros, es una inversión heterogénea, compuesta por activos cuyo rendimiento no está directamente relacionado con la evolución de los mercados financieros cuyo objetivo es el obtener retornos positivos, independientemente de la evolución de los mercados. Los activos no financieros están formados por los activos de colección, los inmuebles, las materias primas, los metales y otros como los recursos naturales. Estas inversiones podrán ayudar a mejorar el binomio rentabilidad-riesgo de las carteras de inversión (López y Hurtado, 2008).

Los activos de colección o bienes de colección son activos no financieros que se engloban dentro de las inversiones no financieras, o complementarias a las financieras. Dentro de los activos de colección se encuentran, las monedas, los sellos, la pintura, la escultura, la obra gráfica, las antigüedades, los libros antiguos y los incunables, los autógrafos, etc. Los activos de colección poseen un valor de cambio en el momento de la venta o en el momento en que un experto lo tasa y le proporciona un valor.

Los activos numismáticos compuestos por las monedas históricas, que son aquellas que han estado o están en circulación, como por ejemplo, los denarios romanos, la peseta, el dólar estadounidense, etc.; las conmemorativas ${ }^{3}$ aquellas que emite un gobierno para conmemorar algún suceso acuñadas en metales preciosos y con tiradas pequeñas; y las monedas bullions, aquellas acuñadas en metales preciosos (oro, plata, platino, paladio) cuyo precio se fija por el metal de acuñación, más una prima de entre el $3 \%$ y el $6 \%$ sobre el precio de ellas.

Las características específicas de un bien de colección (Coca, 1998; 2001a; 2001b), donde se encuentran los activos numismáticos, son las siguientes: Tangibilidad, uno posee en propiedad el valor de su inversión. Valor - refugio, entendemos por él, aquel activo (físico o financiero) que por sus especiales características de oferta y demanda, quedan al margen de las crisis de los mercados financieros así como de las economías reales. Universalidad de los mercados, estos bienes pueden venderse en cualquier parte del mundo y están universalmente aceptados. Accesibilidad, esta es una característica referida a las mondas en particular, prácticamente cualquier inversor/coleccionista tiene suficiente disponibilidad de efectivo para adquirir activos numismáticos. Revalorización, los bienes de colección se revalorizan con el paso del tiempo debido principalmente a la escasez, rareza y antigüedad, produciendo que el valor de este tipo de bienes crezca constantemente. Previsionalidad de ahorro, estas inversiones prevén un ahorro, son inversiones a medio y largo plazo. Aparte de estas seis características comunes, habría que incluir el coleccionismo y la rentabilidad de disfrute puesto que estos bienes son coleccionados y proporcionan una rentabilidad distinta a la económica, la cual es difícil de valorar.

En estos activos, su inversión se caracteriza en: que el precio del activo es muy subjetivo, el activo es único y heterogéneo, la valoración del activo es compleja, durante el tiempo de la inversión no suele generar ningún flujo de caja positivo, esta tiene costes de transacción elevados y son necesarios conocimientos específicos del mercado a la hora de invertir en ellos. Como cualquier otro activo, poseen las características de rentabilidad,

\footnotetext{
${ }^{3}$ La Unión Europea las denomina monedas de colección.
} 
riesgo y liquidez, siendo la liquidez, el principal problema de estos activos, puesto que aunque existen numerosas vías para su comercialización, su conversión en dinero no es inmediata, y dependerá de la situación de su mercado en el momento de la venta.

Además a la hora de invertir es necesario tener en cuenta como restricciones: el horizonte temporal y la fiscalidad del producto. El horizonte temporal es, principalmente, a medio y largo plazo (Salomon Jr y Lennox, 1984; Berman y Schulman, 1986; Coca, 1998, 2001b; Mei y Moses, 2002, 2004; Lombra, 2003; López y Hurtado, 2008), una de las causas son las elevadas comisiones de los intermediarios, por lo que no se pueden aprovechar la descorrelación, a corto plazo, con los mercados tradicionales.

Salomon Jr. y Lennox (1984), destacan que las rentabilidades de los activos tangibles, para periodos de cinco años, eran consistentemente altas, siendo para las monedas americanas de un 9,5\% para el periodo 1969-1974; de un 32,4\% para el periodo 1974-1979 y de un 11,3\% para el periodo 1979-1984. Berman y Schulman (1986), realizan un estudio de las mejores inversiones estadounidenses por beneficio para diferentes periodos. Destacando las monedas históricas, las monedas bullions de oro y de plata, siendo sus rentabilidades de $20,4 \%, 6,9 \%$ y $3,5 \%$ respectivamente para el periodo $1975-1985$, y de $17,7 \%, 15,5 \%$ y $8,7 \%$ para el periodo 1970-1985. Dickie, Delorme y Humphreys (1994), estudian los factores determinantes del precio de la moneda histórica norteamericana y la predicción de este, mediante regresión hedónica con transformación en logaritmos, utilizando como variables el valor facial, el año de emisión, el tipo de imagen, la ceca, el valor de la plata que contienen las monedas y la calidad. Lombra (2003), calcula las rentabilidades medias de los activos numismáticos americanos ${ }^{4}$ siendo las rentabilidades del $14,23 \%$, para las monedas históricas y de un 3,10\%, para las monedas bullion de oro. Prado (2009) realizó un análisis de los factores determinantes en la valoración de los activos numismáticos de oro, mediante modelos de regresión lineal múltiple, obteniendo que el modelo propuesto explicaba entorno al $50 \%$ del precio de estos activos, pudiendo alcanzar hasta un 60\%; obtuvo, mediante un análisis de redes neuronales autoorganizadas, que existen diferencias significativas entre: a) las monedas bullions y conmemorativas (de EEUU y Europa ${ }^{5}$ ), b) las históricas de EEUU, c) las históricas europeas de calidad alta, y d) las de calidad media-baja.

Brown (2005) analiza el activo moneda histórica de EEUU, como activo de inversión para el periodo 1941-2003, obteniendo para el activo numismático analizado, una rentabilidad media anual sin descontar la inflación de un 10,5\%, con una desviación típica del 12,3\%; una rentabilidad media anual descontando la inflación de un 5,52\% y una rentabilidad por unidad de riesgo $0,46 \mathrm{x}$; siendo, para el mismo periodo, de $0,46 \mathrm{x}$ y de 0,18x para el S\&P 500 y los bonos de EEUU a largo plazo. Es importante señalar que, las revalorizaciones medias son mayores a medida que aumenta la calidad de estos activos (Lombra, 2003). Knaus (2006) realiza un estudio sobre la inversión en monedas históricas americanas, llegando a la conclusión que este tipo de activos tienen rentabilidades altas, de un $12,7 \%$ de rentabilidad anual para periodos de 35 años. También explica que para periodos cortos las rentabilidades de estos activos están no correlacionadas con las acciones, protegen contra la inflación, reducen la volatilidad de las carteras y son una inversión

\footnotetext{
${ }^{4}$ Lombra, R. (2003); estudio de carteras para diferentes activos norteamericanos, renta variable americana, renta fija americana, numismática y bullions de oro; para un período de 25 años (1978-2002).

${ }^{5}$ Representada por Gran Bretaña, Francia y España.
} 
socialmente responsable. Prado (2009), calcula las revalorizaciones medias anuales para los activos numismáticos de oro, emitidos por España, Francia, EEUU y Gran Bretaña de 1900-2008 para el periodo 2003-2008 comparándolos con el oro. Siendo el oro, el que obtuvo la mayor revalorización para este periodo, un 19,53\%, y las revalorizaciones para los activos numismáticos de oro ${ }^{6}$ variaban entre un 14,32 para el bullion de EEUU y un -7,44\% para la conmemorativa de España.

El mercado de los bienes de colección es un mercado estructurado, abierto y universal, al igual que otros mercados en los que se negocian productos múltiples. Este mercado ${ }^{7}$ se apoya en varias consideraciones: Su venta se puede hacer en cualquier parte del mundo al ser unos bienes físicos y al estar universalmente aceptados. Su fácil accesibilidad, sobre todo en monedas y sellos, permite que cualquier persona (física o jurídica) pueda invertir en estos bienes. Existe una amplia oferta que emana de diversas fuentes emisoras: los propios artistas / realizadores de obras de arte (pintura, escultura, obra gráfica, etc.) y el propio Estado (numismática, filatelia). La principal fuente de demanda de los bienes de colección proviene de los coleccionistas. Las principales vías de comercialización de estos activos son: comercios especializados, casas de subastas, galerías y los productos de inversión ${ }^{8}$.

El mercado de bienes de colección a nivel mundial se divide entre EEUU con un $46 \%$ de cuota de participación, Reino Unido con un 27\%, Francia con un 6,4\%, China con un $5 \%$, Alemania con un 2,9\% y el resto de países con un $12,7 \%{ }^{9}$ (McAndrew, TEFAF 2008). Respecto al mercado numismático internacional actualmente está en auge, detectándose un aumento de coleccionistas, dado que la frecuencia y calidad de las subastas ha crecido espectacularmente (Prado et al, 2007; Prado, 2009). Es un mercado activo y bien organizado, aseveración avalada por el análisis de los catálogos nacionales e internacionales de casas de subastas, asociaciones y estudios, tales como, Dickie et al, 1994; Brown, 2005, siendo este último, quien utilizando datos de la American Numismatic Association, determinará que el mercado numismático tiene un volumen de negociación, excluyendo monedas de plata y bullions de oro, superior a los $\$ 40$ billones de dólares. En la misma línea y para el mercado de EEUU (Siconolfi, 1990; Dickie et al, 1994 y la Coin World Inc., 2003), se estimó que las ventas anuales en un mercado tan específico como el mercado numismático de moneda histórica de EEUU, rondan los \$5 billones de dólares.

\footnotetext{
${ }^{6}$ Revalorizaciones medias anuales para los activos numismáticos de oro, emitidos por España, Francia, EEUU y Gran Bretaña de 1900-2008 para el periodo 2003-2008: las monedas Bullions de oro de EEUU, la histórica de EEUU y la conmemorativa EEUU obtuvieron un $14,32 \%, 14,27 \%$ y un $11,36 \%$ respectivamente, siendo estas las mayores revalorizaciones. Dentro de las europeas, un 2,44\% para el bullion de Gran Bretaña, un 1,95\% para la histórica de Francia, un 0,13\% para la histórica de Gran Bretaña, un -4,75\% para la conmemorativa de Francia, un $-6,10 \%$ para la histórica de España y un $-7,44 \%$ para la conmemorativa España.

${ }^{7}$ La actividad de los bienes de colección se regula por las leyes de cada país. En España la ley de inversión en bienes tangibles, o LEY 43/2007, de 13 de diciembre, de protección de los consumidores en la contratación de bienes con oferta de restitución del precio, regula la actividad (BOE núm. 299, de 14 de diciembre) y viene a sustituir a la Ley 35/2003, de 4 de noviembre, de Instituciones de Inversión Colectiva (BOE, de 5 de noviembre de 2003).

${ }^{8}$ Los productos de inversión basados en estos bienes se dividen en financieros y no financieros. Dentro de los financieros, los fondos de inversión en monedas bullions de oro (Ever Bank Fund), en arte (Art Collectors Fund, Contemporany Chinese Fund, The Fine Art Fund, entre otros), en obra gráfica (Cannoball Art Fund) o en fotografía (WMG). Dentro de los no financieros, las carteras de inversión de sellos (Stanley Gibbons Group Corp.) o de autógrafos (Fraser's Autographs Corp.).

${ }^{9}$ España representó un 0,6\% de cuota de participación en el 2006 y un 0,7\% en el 2007.
} 


\section{TEORÍA DE CARTERAS}

En las últimas décadas el desarrollo de los mercados financieros y de los productos ofertados en ellos ha crecido de una manera exponencial. Esta circunstancia ha servido de base para el nacimiento de diferentes métodos para gestionar el riesgo inherente a este tipo de inversiones. La base analítica de buena parte de estos métodos se asienta en los estudios de Harry Markowitz $(1952,1959)$ sobre diversificación de las inversiones, donde analiza el problema al que se enfrenta cualquier ahorrador a la hora de colocar sus recursos financieros en el mercado: la obtención de la mayor rentabilidad con el menor riesgo posible.

La rentabilidad de una cartera va a venir determinada por la media ponderada de los diferentes activos que la componen. El riesgo total de la cartera va a depender de tres factores: 1 Las ponderaciones de los activos; 2 Las varianzas de las rentabilidades de cada uno de los activos; y 3 La correlación existente entre cada uno de los activos. Este último es clave para la construcción de las carteras, ya que implica que al aumentar el número de activos en una cartera de inversión la rentabilidad de esta aumentará proporcionalmente a la rentabilidad de dicho activo ponderada por su peso en la cartera, mientras que el riesgo de la misma va a verse afectado por el grado de correlación existente entre todos los activos presentes en ella. Basándose en esta propiedad, Markowitz establece un modelo de gestión de carteras consistente en optimizar un sistema de ecuaciones basado en la rentabilidad y en el riesgo de la cartera. Fruto de la maximización de la función de rentabilidad de la cartera obtendremos la curva que recoge todas aquellas carteras eficientes existentes en el mercado y al obtener el punto de corte de esta curva con la curva de aversión al riesgo del inversor, que se puede describir como una función de utilidad, nos proporcionará aquella combinación de activos que posee el mejor binomio rentabilidad / riesgo acorde a las preferencias del inversor. Demostrándose que al combinar activos de diferente naturaleza, se podía reducir el riesgo total de la cartera, gracias a la incidencia de la correlación entre activos a la hora de calcular la varianza total de la cartera.

Las conclusiones alcanzadas por Markowitz en el desarrollo de su teoría han resultado de suma utilidad, tanto en el plano académico como en el plano profesional. Aunque al principio la complejidad matemática del método produjo que su utilización fuese menor, por lo que Sharpe (1964) planteó una simplificación basada en la existencia de una relación lineal entre el rendimiento del título y el de la cartera de mercado, actualmente se dispone de las herramientas necesarias para su cálculo. También propusieron simplificaciones, Elton et al (1976; 1978) y Konno y Yamakazi (1992), aunque se demostró que estas no eran óptimas (Burgess y Bey, 1988; Roll, 1992; Simaan, 1997).

El modelo de Markovitz no tiene en cuenta los costes de transacción ni los impuestos, considera la perfecta divisibilidad de los títulos escogidos, además el inversor tiene dificultades en valorar su actitud ante el riesgo y deducir su función de utilidad (Michaud, 1989; Frankfurter y Phillips, 1995). Esta técnica tiene una serie de ventajas, destacando, control de la exposición de la cartera al riesgo, restricciones de los inversores, satisfacción de los objetivos, establecimiento del estilo de inversión, uso eficiente de la información (Michaud, 1989). No obstante, es destacable el hecho de que los niveles de riesgo pueden ser reducidos de manera importante si la diversificación es realizada de la manera adecuada, esto es, si las inversiones que compren las carteras están poco correlacionadas entre sí. Evidencia de ello se observa al analizar los efectos que la inversión internacional puede tener en la rentabilidad y riesgo de las carteras de valores (Solnik, 1974). 
La existencia de riesgos no diversificables que afectan a los activos financieros, aunque en distinto grado, proporcionan al inversor la "tarea" de encontrar activos acordes a su perfil de riesgo mediante una adecuada gestión del riesgo. Algunos estudios hacen hincapié en el carácter estable de inversiones como son la filatélica, numismática o, en definitiva, la inversión en activos tangibles de colección (Sharpe, 1963; 1964). En esta línea, Sharpe y Alexander (1990), califican los "activos tangibles", como activos que sirven para cubrirse contra la inflación mejor que otros como las acciones o los bonos.

Randall (1981), estudia la inversión en bienes de colección en épocas inflacionistas partiendo del supuesto que existe una cartera de tipo de interés bajista que paga un $3 \%$ sobre el ratio de inflación, obteniendo que la cartera compuesta por activos tangibles se aprecia un $1 \%$ al año más rápido que el ratio de inflación. Barr y Affleck-Graves (1985), estudian las vías de inversión en oro, creando carteras con bullions de oro y otras con lingotes, llegando a la conclusión que la inversión en lingotes históricamente en mejor que en bullions de oro. Flôres Jr. et al (1999) compararon carteras de obras de pintura para el corto y el largo plazo; basándose en que los precios del pintor conocido y del pintor no conocido se mueven a la vez, en las tres plazas más importantes (Londres, Paris y New York) y en que los inversores son indiferentes entre los dos grupos de artistas. Demostraron que el pintor conocido tiene menor riesgo que aquel no contrastado; y el porcentaje de artista consagrado en las carteras debía de ser superior al $90 \%$.

Lombra (2003; 2004), realiza un análisis econométrico sobre el comportamiento de las carteras de inversión que incluían tanto numismática estadounidense como bullions de oro (compuesto por monedas bullions y lingotes y barras), respecto a carteras que excluían estos activos. De la Poza et al (2008) realizan un estudio sobre diversificación de carteras con activos financieros (renta variable) y activos de colección (pintura), demostrando que combinando los activos de colección y la renta variable, un inversor obtiene mayores retornos con el mismo nivel de riesgo; también demuestra, que el inversor en arte reduce la volatilidad de su cartera al introducir la pintura en una cartera de renta variable, sirviendo como diversificadores del riesgo. Otros estudios, Capgemini y Merrill Lynch (2007), señalan que los gestores de patrimonio tienen en cuenta este mercado "están viendo la pintura, los dibujos o la escultura, como un vehículo para diversificar sus carteras dada la baja correlación existente entre los precios del arte y los mercados cíclicos como las acciones o el inmobiliario".

\section{DESCRIPCIÓN DE LA MUESTRA Y LA METODOLOGÍA}

La muestra empleada para el análisis está compuesta por: los metales preciosos (oro, plata, platino) más los metales paladio y rodio, y los activos numismáticos de oro, en sus tres tipos: monedas históricas, monedas conmemorativas y monedas bullions, emitidos por España, EEUU, Gran Bretaña y Francia, desde 1900 hasta el 2009, para el período 2003-2009.

El catálogo utilizado para conocer los diferentes valores de mercado de los activos numismáticos de oro, así como las características de estos activos de inversión, es el Standard Catalogue of World Coin ${ }^{10}$. Catálogo reconocido internacionalmente por expertos numis-

\footnotetext{
${ }^{10}$ El catálogo utilizado, el Standard Catalogue of World Coin, es editado en EEUU y es aceptado por coleccionistas y expertos numismáticos.
} 
máticos. Los años seleccionado para el estudio han sido 2003, 2004, 2005, 2006, 2007, 2008 y 2009, para todos los países. Los valores de los metales (oro, plata, platino, paladio y rodio) se han obtenido a partir de la base de datos de Kitco.com.

Los activos numismáticos de oro que componen las diferentes carteras para el periodo 2003-2009 son: el oro, la plata y el platino (metales preciosos), el paladio y el rodio (todos dentro de los metales nobles), y los activos numismáticos de oro a partir de su clasificación (Prado, 2009), estos son, moneda histórica de EEUU de calidad alta y de calidad baja, moneda histórica de Gran Bretaña de calidad alta y baja, moneda histórica de Francia de calidad alta y baja, moneda histórica de España de calidad alta y baja, moneda bullion de EEUU de calidad alta, moneda bullion de Gran Bretaña de calidad alta y de calidad baja, moneda conmemorativa de EEUU de calidad alta y baja, moneda conmemorativa de Francia de calidad alta y moneda conmemorativa de España de calidad alta ${ }^{11}$.

La metodología que utilizamos es el modelo matemático realizado por Markowitz, que plasma el comportamiento de los inversores. Los inversores quieren maximizar su rendimiento manteniendo el riesgo constante (1); o minimizar el riesgo manteniendo el rendimiento constante (2). Matemáticamente se escribiría como (1):

$$
\begin{gathered}
\left.\operatorname{Max} \amalg E(R]_{p}\right)=\sum_{i=1}^{n} x_{i} E\left(R_{i}\right) \\
\sigma^{2}\left(R_{p}\right)=\sum_{i=1}^{n} \sum_{i=j}^{n} x_{i} x_{j} \sigma_{i j}=\text { constante } \\
\sum_{i=1}^{n} x_{i}=1 \\
x_{i} \geq 0(i=1, \ldots, n)
\end{gathered}
$$

También se podría escribir como (2):

$$
\begin{gathered}
\text { Min } \sigma^{2}\left(R_{p}\right)=\sum_{i=1}^{n} \sum_{i=j}^{n} x_{i} x_{j} \sigma_{i j} \\
E\left(R_{p}\right)=\sum_{i=1}^{n} x_{i} E\left(R_{i}\right)=\text { constante } \\
\sum_{i=1}^{n} x_{i}=1
\end{gathered}
$$

\footnotetext{
${ }^{11}$ La calidad alta, en los activos europeos corresponde a la calidad Value, Proof, Unc y BU y en los activos de EEUU a Value, Prf-65, MS-65, MS-64, MS-63 y MS-60. La calidad baja, en los activos europeos corresponde a la calidad XF, EF, VF y F y en los activos de EEUU a AU-50, XF-40, VF-20 y F-12.
} 


$$
x_{i} \geq 0(i=1, \ldots, n)
$$

Siendo las incógnitas, las ponderaciones individuales de los títulos $\left(\mathrm{x}_{\mathrm{i}}\right) ; \sigma^{2}\left(\mathrm{R}_{\mathrm{p}}\right)$ la varianza de la cartera $p$, y $\sigma_{\mathrm{ij}}$ la covarianza entre los rendimientos de los valores $i$ y $j . \mathrm{E}\left(\mathrm{R}_{\mathrm{p}}\right)$ es el rendimiento esperado de la cartera $p$. Al resolverlo y variar la constante, obtendremos el conjunto de ponderaciones que maximizan el rendimiento de la cartera (1), o el conjunto de ponderaciones que minimizan el riesgo de la cartera. La solución es el tanto por ciento que se destina del capital para formar cada una de las carteras del conjunto de soluciones. El conjunto de combinaciones rentabilidad-riesgo, $\left[\mathrm{E}\left(\mathrm{R}_{\mathrm{p}}\right), \sigma^{2}\left(\mathrm{R}_{\mathrm{p}}\right)\right]$, representan las combinaciones posibles de las carteras en el mercado, pero no todas son eficientes, entendiendo por eficiencia la posibilidad de obtener más rendimiento para un mismo riesgo, o menos riesgo para un mismo rendimiento. Se puede demostrar que las carteras más eficientes son aquellas que se sitúan en el borde superior izquierdo del conjunto que compone la nube de puntos. Ese borde, que se traza con una línea continua, se denomina la Frontera Eficiente, combinaciones rentabilidad-riesgo de las carteras eficientes.

Al añadir los bonos del Tesoro, activos denominados Libre de Riesgo, puesto que su rendimiento no depende de la variabilidad de los rendimientos históricos. Se deduce que existirá siempre un punto en la Frontera Eficiente que al combinarlo con el activo libre de riesgo formará una cartera superior a las demás. A este punto se le denomina la Cartera del Mercado y su combinación con el activo libre de riesgo forma una línea que ofrece, para cualquier riesgo, más rendimiento que cualquier otra combinación del mercado. A esta línea se le llama Línea del Mercado de Capitales o CML. Cuando hemos incluido en nuestro modelo la posibilidad de invertir en el activo libre de riesgo la eficiencia pasa de una curva, la frontera, a una recta, la CML. La ecuación que explica la CML es la siguiente:

$$
E_{p}=R f+\frac{E_{M}-R f}{\sigma_{M}} \cdot \sigma_{p}
$$

Esta ecuación señala que el rendimiento de una cartera es, como mínimo, el rendimiento del activo libre de riesgo $(R f)$, y este rendimiento se irá incrementando en función de asumir más riesgo en la cartera $\left(\sigma_{p}\right)$. Por cada unidad de riesgo asumida el mercado pagará una prima de riesgo, $\left[\left(E_{M}-R f\right) / \sigma_{M}\right]$.

Se ha considerado la restricción de no negatividad de los pesos puesto que nos estamos centrando en activos no financieros. Las ventas a corto no son contempladas puesto que al ser bienes tangibles, en el momento de su venta se ha de disponer del bien.

El programa informático utilizado es La Toolkit financiera del MatLab, la cual nos permite calcular la frontera eficiente, maximizando el rendimiento para los riesgos dados.

\section{ANÁLISIS DE RESULTADOS}

Los activos numismáticos de oro que componen las diferentes carteras para el periodo 2003-2009 son: dentro de los metales nobles: el oro (1), la plata (17), el platino (18), el paladio (19) y el rodio (20) y dentro de los activos numismáticos de oro a partir de su clasificación, estos son, moneda histórica de EEUU de calidad alta (2) y de calidad baja 
(3), moneda histórica de Gran Bretaña de calidad alta (8) y baja (4), moneda histórica de Francia de calidad alta (7) y baja (5), moneda histórica de España de calidad alta (9) y baja (6), moneda bullion de EEUU de calidad alta (10), moneda bullion de Gran Bretaña de calidad alta (13) y de calidad baja (14), moneda conmemorativa de EEUU de calidad alta (16) y baja (11), moneda conmemorativa de Francia de calidad alta (15) y moneda conmemorativa de España de calidad alta (12).

Con los datos utilizados se calcula las correlaciones entre los diferentes activos de inversión (Ver Anexo, tabla 3). Los datos aportados muestran una gran correlación entre ellos. Esto provoca que se trabaje con altas covarianzas afectando al resultado del trabajo. Entendiendo que los inversores, en aras de reducir su riesgo no elegirían carteras muy correlacionadas, se ha procedido a quitar del modelo aquellos productos con correlaciones mayores a 0,8 . Las correlaciones negativas, aun siendo altas en valor absoluto, reducen el riesgo de la cartera, por lo que se han mantenido. Estos son, el oro; la moneda histórica de EEUU de calidad alta; la moneda histórica de EEUU de calidad baja; la moneda histórica de Gran Bretaña de calidad baja; la moneda histórica de Francia de calidad baja; la moneda histórica de Francia de calidad alta; la moneda bullion de EEUU de calidad alta; la moneda conmemorativa de EEUU de calidad baja y el rodio. En la Tabla 4 del Anexo podemos ver las correlaciones de estos activos. En la Tabla 1 podemos ver la matriz de varianzas y covarianzas de los activos del modelo y el vector de las esperanzas matemáticas de los rendimientos de los activos del modelo.

Tabla 1

Matriz de varianzas (sombreadas) y covarianzas y las esperanzas matemáticas de los rendimientos de los activos del modelo

\begin{tabular}{|c|c|c|c|c|c|c|c|c|c|}
\hline & $\mathrm{Or}$ & $\begin{array}{l}\text { Merarcet } \\
\text { cotas. }\end{array}$ & 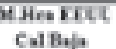 & $\begin{array}{l}\text { MHes CB } \\
\text { Catsojs }\end{array}$ & $\begin{array}{c}\text { Mhancals } \\
\text { Bets }\end{array}$ & $\begin{array}{l}\text { Hew Fral } \\
\text { Als. }\end{array}$ & 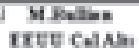 & 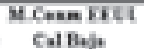 & Dode \\
\hline 00 & Q.9:7 & Depe & 4.5004 & 0,0004 & 4.0544 & 0.0006 & $4 \sec 4$ & 0.179 & 0,000 \\
\hline $\begin{array}{l}\text { SA Hes ExEE } \\
\text { Calas }\end{array}$ & 0.0042 & o,ects & antos & $0,003 i$ & 2.0015 & $0,000 s$ & a,seto & 0,0000 & $0,000=$ \\
\hline $\begin{array}{l}\text { MHarirev } \\
\text { CalBofe }\end{array}$ & eseces & 6,6064 & anass & Acois & taxt7 & osti1 & $4, x \in$ & Aessi & $-9,0000$ \\
\hline $\begin{array}{l}\text { Mine ce Cal } \\
\text { Dope }\end{array}$ & 0,004 & $-4,0003$ & 4.xets & 0,0007 & 4.253 & Q.00: & Q.xan & 0,000 & $0.006 x$ \\
\hline $\begin{array}{l}\text { MHesfreal } \\
\text { Bupe }\end{array}$ & 0.0054 & botous & 0.027 & 0,0013 & $2,00 \times 0$ & 0.0004 & 0.0257 & e.00322 & $-0,000=$ \\
\hline $\begin{array}{l}\text { Milla FrCal } \\
\text { Alus }\end{array}$ & 0,0006 & 6,0005 & atus & 0.0011 & 4,005 & oens & $4.505 ?$ & acoss & ateis \\
\hline 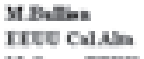 & 40014 & Q.eols & Axest & 0.003 & s.0es7 & 4 Dost & Q.1.46 & 0,0004 & D.PUER \\
\hline $\begin{array}{l}\text { M.Cum EXEE } \\
\text { CalBula }\end{array}$ & 0.010 & $-0,0006$ & s.t.esi & 0,000 & $-0,0 \times 12$ & 0,00ts & 0,0001 & 0,0210 & $0,015 \mathrm{~s}$ \\
\hline Redis & Anes & - atest & $48 \times 5$ & eneses & 4.000 & Aster & $\operatorname{sen}$ & osts & $\operatorname{sen} 2$ \\
\hline Mendie & Q.1987 & 6.0003 & $2 \times 04$ & - 0.0013 & 2.0013 & 40003 & ant? & 0.138 & 0.454 \\
\hline
\end{tabular}

Se ha dicho que el objetivo del trabajo es la construcción de diferentes carteras compuestas por activos numismáticos de oro, más los metales (oro, plata, platino, paladio y rodio). Con el fin de construir aquella cartera que mejor se adapte al inversor acorde a su perfil inversor y conocer cuál es la Cartera del Mercado, mediante el uso de la Teoría de Carteras, construiremos la Frontera Eficiente y trazaremos la Línea del Mercado de Capi- 
tales o CML. Para ello en un primer momento hemos simulando 50.000 carteras aleatorias que nos permita dibujar el universo inverso, obteniendo la siguiente figura.

Figura 1

\section{Simulación de $\mathbf{5 0 . 0 0 0}$ carteras con los nueve activos seleccionados}

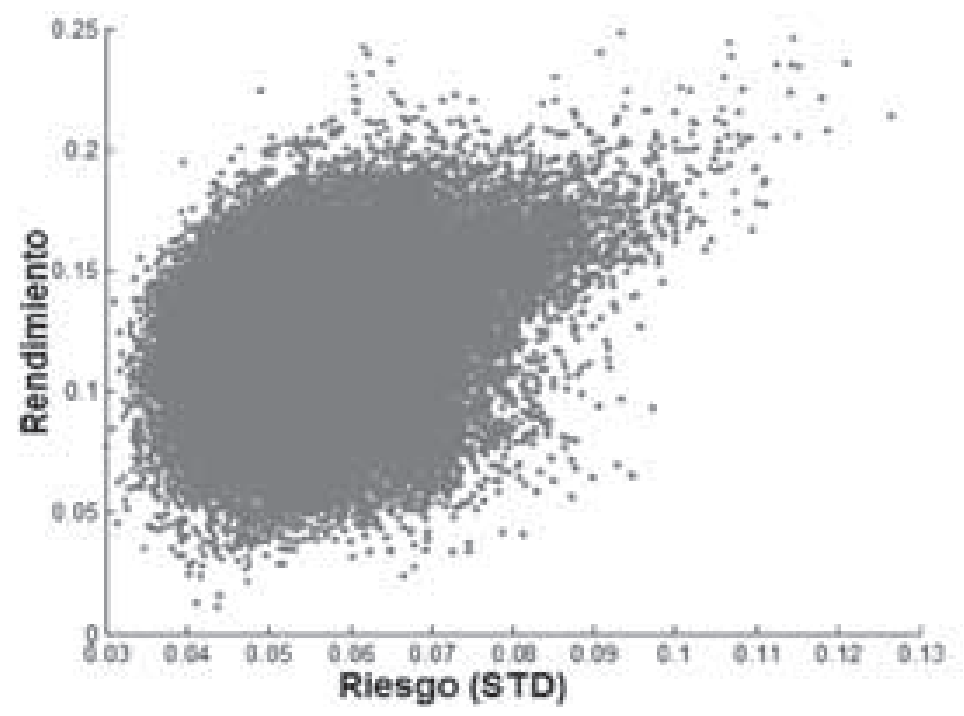

Como se puede observar en la figura 1 se obtiene una nube de puntos con una forma convexa, desde el eje de ordenadas, muy parecida al que se forman en los mercados de acciones. Sin embargo, hay que decir que si el modelo se hubiera realizado con todos los productos asumiendo su alta correlación y por tanto sus altas covarianzas, la nube hubiese perdido su forma curva asemejándose más a una recta de puntos (Suárez, 2004).

El programa informático utilizado (La Toolkit financiera del MatLab) permite calcular la frontera eficiente, no uniendo los puntos más alejados de la nube de puntos, sino maximizando el rendimiento para los riesgos dados. Optimizando de esta manera obtendremos los pesos de cada una de las nueve alternativas que maximizan los rendimientos sin modificar el riesgo. Con este sistema se obtienen 50 carteras de la Frontera Eficiente. Los resultados detallados se muestran en el Anexo, tabla 5.

En la Figura 2 se muestra la composición de las cincuenta carteras que componen la Frontera Eficiente en un gráfico de líneas. Cada una de las líneas representa el tanto por ciento de inversión en cada producto. Como se puede observar, las carteras que ofrecen mayor rendimiento, y por tanto mayor riesgo, son las que invierten casi toda su renta en el Rodio; mientras que las carteras con menor rendimiento y riesgo están más diversificadas, pues invierten en un conjunto de productos diferentes. 
Figura 2

\section{Composición de las carteras de la Frontera Eficiente}

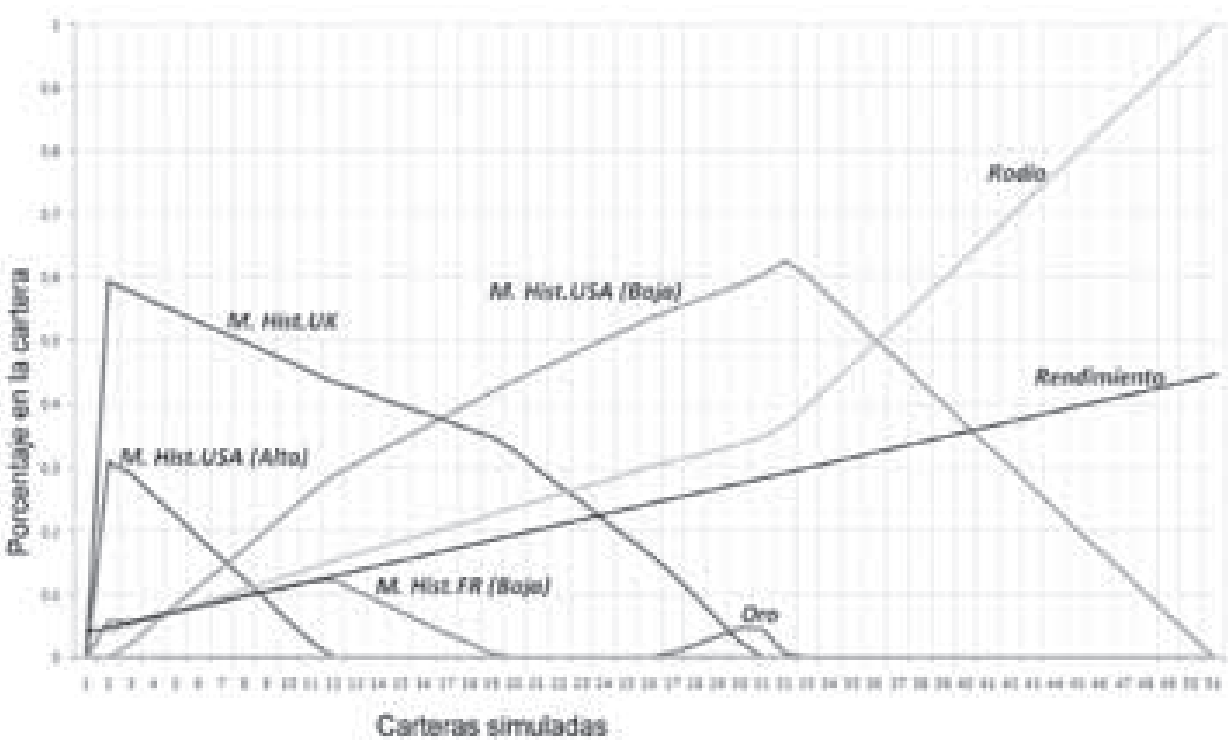

Con esos datos podemos dibujar la Frontera Eficiente, que mostramos en la Figura 3.

Figura 3

Frontera Eficiente del modelo

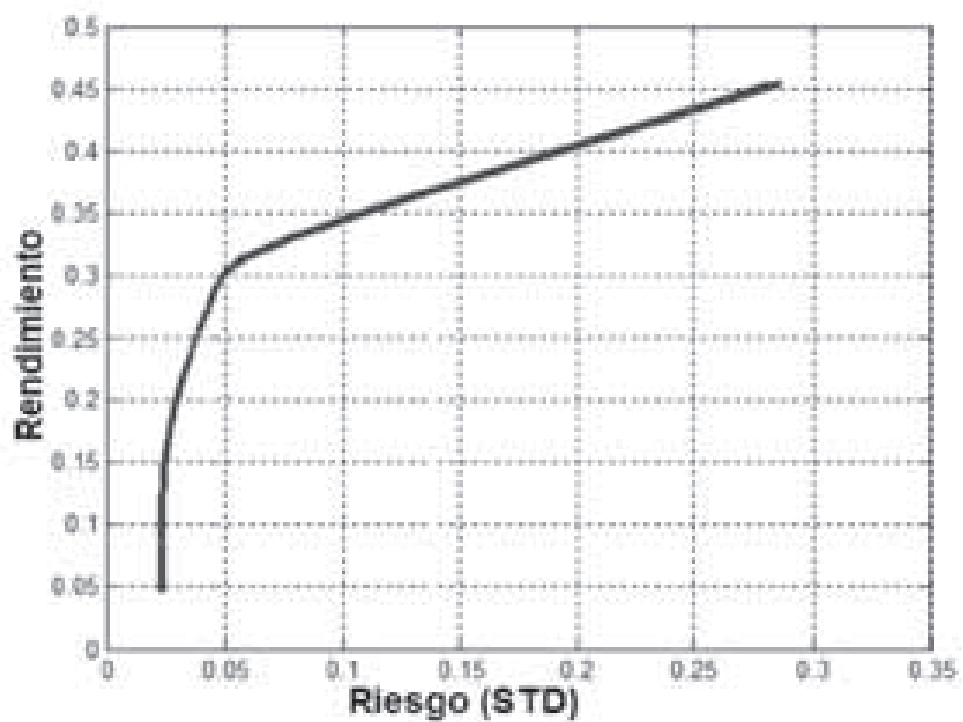


Si asumimos un rendimiento libre de riesgo del $3 \%$ la cartera de mercado se situará en la siguiente inversión:

Tabla 3

Cartera de Mercado para un $\mathrm{R} f$ de $3 \%$

\begin{tabular}{|c|c|c|c|c|c|c|c|c|c|c|}
\hline \multicolumn{9}{|c|}{ 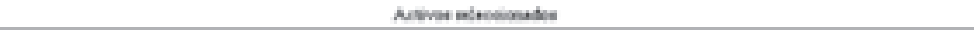 } & \multirow[b]{2}{*}{ Bentrimeso } & \multirow[b]{2}{*}{ Ance } \\
\hline Oro & $\begin{array}{l}\text { MAnstuv } \\
\text { Cedsed es }\end{array}$ & 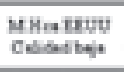 & 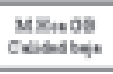 & 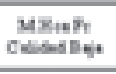 & $\begin{array}{l}\text { MHeahn } \\
\text { Clised ats }\end{array}$ & 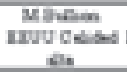 & $\begin{array}{c}\text { Mene } \\
\text { rudCedet } \\
\text { tes. }\end{array}$ & Rede & & \\
\hline o.,ow & e.eots & $30.25 \%$ & $31,70 \mathrm{~s}$ & $0.0 \mathrm{k}$ & asos & axt & ext & mests & nust & $1 \mathrm{sen}$ \\
\hline
\end{tabular}

La cartera de mercado corresponde a una cartera que tiene un rendimiento esperado de $23,05 \%$ anual con una desviación típica de 3,49\%. Cualquier cartera puede ser replicada combinando $R f$ con la cartera de mercado. Se obtiene el mismo rendimiento pero con menos riesgo, pues la teoría nos dice que en la CML no se tiene riesgo propio; sólo sistemático. Es decir, invirtiendo un 50,28\% en moneda histórica americana de calidad baja, $21,76 \%$ en histórica del Gran Bretaña de calidad baja y un 27,96\% en Rodio, maximizamos el rendimiento hasta $23,05 \%$ soportando un riesgo del 3,49\%.

Figura 4

Cartera eficiente para un interés libre de riesgo del $3 \%$

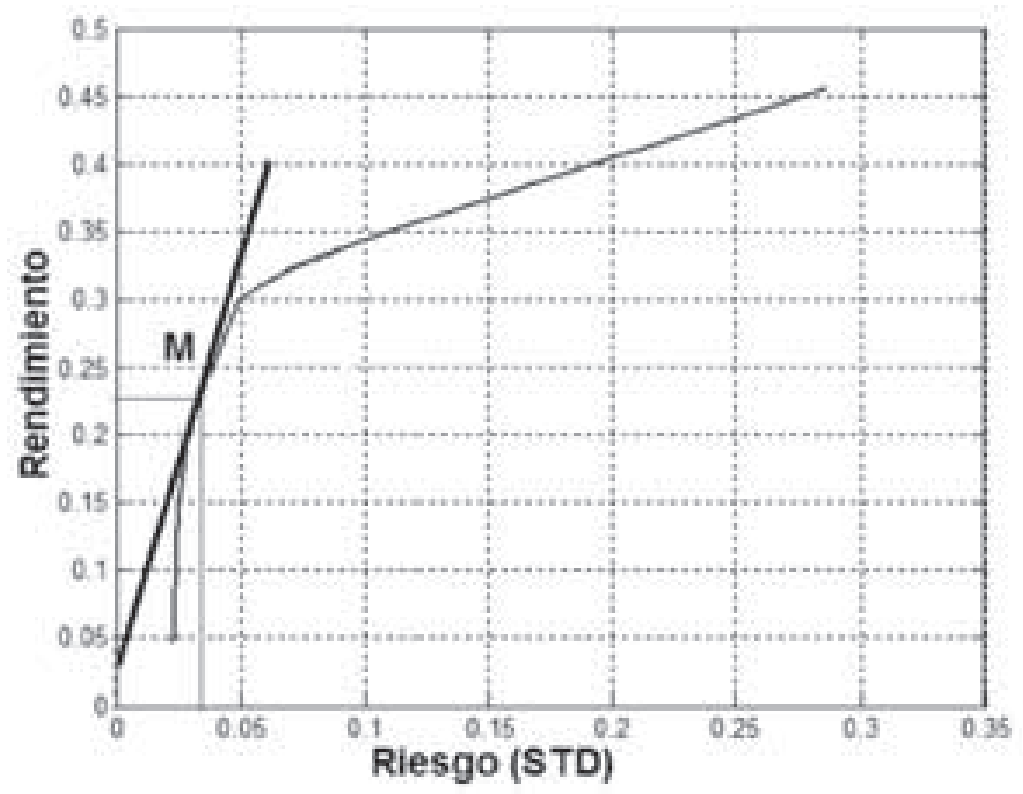


Los inversores deben establecer el riesgo que quieren soportar y calcular cuál es la proporción entre el rendimiento libre de riesgo y la cartera de mercado que les sitúa en la Frontera Eficiente. Cualquier combinación que nos sitúe en esta recta, siempre nos ofrecerá una combinación de rendimiento riesgo mejor que cualquiera de los puntos de las 50.000 carteras dibujadas.

\section{CONCLUSIONES}

La Teoría de Carteras nos ofrece la posibilidad de una mejor gestión del riesgo, presente en el mercado numismático y en el mercado del oro. Los Bullion son activos de colección interesantes para la inversión por su gran liquidez y buena rentabilidad, conllevan rigideces ocasionadas por la imposibilidad de gestionar el riesgo intrínseco derivado de poseer características de dos mercados diferenciados como son el numismático y el del oro. Una cartera compuesta por monedas históricas y oro nos reporta las bondades de ambos mercado, permitiéndonos además la gestión del riesgo intrínseco en ellos, en contra punto a los bullion o monedas conmemorativas.

En la construcción de carteras, no todos los activos seleccionados son utilizados para construir la cartera eficiente. Los que han sido utilizados, son el oro y el rodio, como metales nobles; casi la totalidad de los activos numismáticos emitidos por EEUU (monedas históricas de calidad alta y baja; bullions de calidad alta; y monedas conmemorativas de calidad alta). Dentro de Gran Bretaña las monedas históricas de calidad baja, y dentro de Francia las históricas de calidad alta y de calidad baja. De España no ha sido seleccionado ningún activo.

La cartera de mercado corresponde a una cartera que tiene un rendimiento esperado de $23,05 \%$ anual con un riesgo de 3,49\%. Compuesta por un 50,28\% en moneda histórica de EEUU de calidad baja, 21,76\% en histórica Gran Bretaña de calidad baja y un 27,96\% en rodio.

Las carteras construidas, son carteras compuestas por pocos activos. A mayor riesgo menor diversificación de la cartera, destacando que una cartera con altos niveles de riesgo $28,68 \%$ y un de rendimiento $45,55 \%$, está compuesta en un $100 \%$ por rodio (cartera número 50).

Por contra para riesgo bajo se construyen carteras con un mayor número de activos. Para la cartera número 1 , cartera con un riesgo de $2,27 \%$ y rendimiento $4,71 \%$, la composición de activos es la siguiente, monedas históricas de EEUU de calidad alta (30,95\%), monedas históricas de Gran Bretaña de calidad baja (59,33\%), moneda histórica de Francia de calidad baja $(5,79 \%)$ y rodio $(3,93 \%)$. Al aumentar el riesgo (cartera número 26 ) a un $4 \%$, con rendimiento $25,55 \%$, la cartera está compuesta por el oro $(0,74 \%)$, el rodio $(31,06 \%)$, la moneda histórica de EEUU de calidad baja $(55,02 \%)$ y monedas históricas de Gran Bretaña de calidad baja (13,18\%).

Las inversiones en activos no financieros, al no pertenecer al mercado financiero, pueden utilizarse como diversificadores del riesgo. Al construir carteras compuestas por activos numismáticos de oro y metales nobles se observa que los activos numismáticos de EEUU son los más utilizados y dentro de los metales nobles el oro y el rodio. 


\subsection{Limitaciones y futuras líneas}

El presente estudio pretende la construcción de diferentes carteras compuestas por activos numismáticos de oro y metales nobles, aunque presenta limitaciones. Aunque los cuatro países objeto de estudio representan más del $80 \%$ de cuota de participación en el mercado de bienes de colección a nivel mundial, sería necesario incluir algún país de Asia (como por ejemplo China, que representa actualmente un 5\%) para tener una visión más global de este mercado. También es cierto, que con respecto al mercado europeo, hemos seleccionado, tres países objeto de estudio que representan más del 34\% de cuota de participación a nivel mundial. Por lo que deberíamos incluir otros países europeos con peso en dicho mercado, como son Italia o Alemania, entre otros.

Deberíamos ampliar el número de años estudiados, recordemos que el periodo de estudio es del 2003 al 2009. Aunque los datos objeto de estudio son las emisiones desde 1900 a 2009, de Gran Bretaña, España, EEUU y Francia. Se podría ampliar el número de activos numismáticos, es decir, todas aquellas monedas que por su valor histórico, su antigüedad, escasez, etc., son objeto de valoración por los coleccionistas y/o inversores, ya sean en oro o en otros metales. Y ampliar a otros metales preciosos el estudio de las monedas bullions (vía de inversión accesible al inversor medio en metales preciosos).

Por último, como hemos dicho, se podría ampliar los activos seleccionados para la construcción de carteras, así como, su introducción en carteras de inversión compuestas por productos financieros.

\section{REFERENCIAS BIBLIOGRAFÍCAS}

BARR, G.D.I. y AFFLECK-GRAVES, J.F. (1985): "Gold shares or gold bullion, which is the better investment?", Managerial and Decision Economics, Vol. 6, № 4, pp. 241-246.

BERMAN, N.S. y SCHULMAN, H.M.F. (1986): The Investor's Guide to United States Coins, Coins and Currency Institute, United States of America.

BOLSA Y MERCADOS ESPAÑOLES, ABANTE Y CINCO DÍAS (2007): Guía práctica de inversión, Biblioteca empresarial Cinco Días, Madrid.

BROWN, R.A. (2005): "Rare Coins: A Distinct and Attractive Asset Class", Journal of Financial Planning, http://www.fpanet.org/journal/BetweenTheIssues/Contributions/080105.cfm, (mayo 2009).

BRUCE, I.I.; COLIN, R. y THOMAS, M. (2006): 2006 Standard Catalog of World Coins, based on the original work by Chester L. Krause y Clifford Mishler, 33rd edition, KP, USA.

BRUCE, I.I.; COLIN, R. y THOMAS, M. (2007): 2007 Standard Catalog of World Coins, 1901 2000, based on the original work by Chester L. Krause y Clifford Mishler, 34th edition, KP, USA.

BRUCE, I.I.; COLIN, R. y THOMAS, M. (2007): 2007 Standard Catalog of World Coins, 2001 Date, based on the original work by Chester L. Krause y Clifford Mishler, 1st edition, KP, USA.

BRUCE, I.I.; COLIN, R. y THOMAS, M. (2008): 2008 Standard Catalog of World Coins, 19012000, based on the original work by Chester L. Krause y Clifford Mishler, 35th edition, KP, USA.

BRUCE, I.I.; COLIN, R. y THOMAS, M. (2008): 2008 Standard Catalog of World Coins, 2001 Date, based on the original work by Chester L. Krause y Clifford Mishler, 2nd edition, KP, USA. 
BRUCE, I.I.; COLIN, R. y THOMAS, M. (2009): 2009 Standard Catalog of World Coins, 19012000, based on the original work by Chester L. Krause y Clifford Mishler, 36th edition, KP, USA.

BRUCE, I.I.; COLIN, R. y THOMAS, M. (2009): 2009 Standard Catalog of World Coins, 2001Date, based on the original work by Chester L. Krause y Clifford Mishler, 3rd edition, KP, USA.

BRUCE, I.I.; COLIN, R. y THOMAS, M. (2010): 2010 Standard Catalog of World Coins, 19012000, based on the original work by Chester L. Krause y Clifford Mishler, 37th edition, KP, USA.

BRUCE, I.I.; COLIN, R. y THOMAS, M. (2010): 2010 Standard Catalog of World Coins, 2001Date, based on the original work by Chester L. Krause y Clifford Mishler, 4th edition, KP, USA.

BURGESS, R.C. y BEY, R.P. (1998): "Optimal portfolios: Markowitz full covariance versus simple selection rules", The Journal of Financial Research, Vol. XI, pp. 153-163.

CAPGEMINI y MERRILL LYNCH (2007): World Wealth Report, 2007.

COCA PÉREZ, J.L. (1998): Análisis del mercado financiero de bienes tangibles: El caso particular de la filatelia financiera, Tesis Doctoral, Universidad Complutense de Madrid, Madrid.

COCA PÉREZ, J.L. (2001a): La inversión en bienes tangibles de colección, cien preguntas clave y sus repuestas, Dykinson, Madrid.

COCA PÉREZ, J.L. (2001b): "La inversión en filatelia: organización del mercado y agentes Participantes"; Revista Europea de Dirección y Economía de la Empresa, Vol.10, № 4, pp.21-28.

COIN WORLD INC., www.coinworld.com. (ENERO 2008).

DE LA POZA, E.; GUADALAJARA, N. y MOYA, I. (2008): "Portfolio Management with Art Assets", BRY Building Bridges in a Global Economy, Publication of IABD \& AEDEM, Vol. 15, $\mathrm{N}^{\circ} 3$, pp. 20-26.

DICKIE, M.; DELORME JR., C.D. y HUMPHREYS, J.M. (1994): "Price Determination for a Collectible Good: The Case of Rare U.S. Coins”, Southern Economic Journal, Vol. 61, No 1, pp. 40-51.

ELTON, E.J.; GRUBER, M.J. y PADBERG, M.W. (1976): "Simple criteria for optimal portfolio selection" The Journal of Finance, $\mathrm{N}^{\circ}$ 31, pp. 1341-1357.

ELTON, E.J.; GRUBER, M.J. y PADBERG, M.W. (1978): "Simple criteria for optimal portfolio selection: tracing out the efficient frontier" The Journal of Finance, $\mathrm{N}^{\circ} 33$, pp. 296-302.

FLÔRES JR., R.G.; GINSBURGH, V. y JEANFILS, P. (1999): "Long- and Short-Term Portfolio Choices of Paintings", Journal of Cultural Economics, Vol. 23, pp. 193-210.

FRANKFURTER, G.M. y PHILLIPS, H.E. (1995): Forty years of normative portfolio theory: Isuess, controversies and misconceptions, JAI Press, USA.

JEFFREY, M.C. (2000): "Silver, Platinum \& Palladium Market \& Outlook", CPM Group Report, CPM Group, USA.

KITCO METALS INC., Precious Metal Database: http://www.kitco.com/, (OCTUBRE 2009).

KNAUS, G. (2006): "Rare coins: The new value investment?", Numismatic Investments Corp. Report, Numismatic Investments Corp., http://www.coinportfolios.com/index.html, (octubre 2008).

KONNO, H. y YAMAZAKI, H. (1992): "Mean-absolute deviation portfolio optimization model and its applications to Tokyo stock market" Management Science, $\mathrm{N}^{\circ} 39$, pp. 519-531.

KRAUSE, C.L. y MISHLER, C. (2003): 2003 Standard Catalog of World Coins, 1901-Present, 30st edition, KP, USA.

KRAUSE, C.L. y MISHLER, C. (2003): Standard Catalog of World Coins: World Coin Listing by Date and Mint, $16^{\mathrm{a}}$ Century edition, KP, USA.

KRAUSE, C.L. y MISHLER, C. (2004): 2004 Standard Catalog of World Coins, 1901-Present, 31st edition, KP, USA.

KRAUSE, C.L. y MISHLER, C. (2004): Standard Catalog of World Coins: World Coin Listing by Date and Mint, $17^{\mathrm{a}}$ Century edition, KP, USA. 
KRAUSE, C.L. y MISHLER, C. (2005): 2005 Standard Catalog of World Coins, 1901-Present, 32 edition, KP, USA.

KRAUSE, C.L. y MISHLER, C. (2005): Standard Catalog of World Coins: World Coin Listing by Date and Mint, $18^{\text {a }}$ Century edition, KP, USA.

KRAUSE, C.L. y MISHLER, C. (2006): Standard Catalog of World Coins: World Coin Listing by Date and Mint, $19^{\text {a }}$ Century edition, KP, USA.

LEY 43/2007, de 13 de diciembre, de protección de los consumidores en la contratación de bienes con oferta de restitución del precio, (BOE, $\mathrm{N}^{\circ} 299$, de 14 de diciembre).

LOMBRA, R.E. (2003): The Investment Performance of Rare U.S. Coins, PhD, Penn State University, Pennsylvania, USA.

LOMBRA, R.E. (2004): "Managing Portfolio Risk: The Role of Rare Coins", Penn State University \& U.S. Tangible Investment Corporation Working Paper, United States.

LÓPEZ, F. y HURTADO, R. (2008): Inversiones alternativas: otras formas de gestionar la rentabilidad, Editorial Especial Directivos, Madrid.

MCANDREW, C. (2008): "The International Art Market, A Survey of Europe in a Global Context" The European Fine Art Foundation Report, TEFAF. Maastricht.

MARKOWITZ, H. (1952): "Portfolio Selection”, Journal of Finance, Vol. 7, № 1, pp. 77-91.

MARKOWITZ, H. (1959): Portfolio Selection: Efficient diversification of investments, John Wilwy \& Sons, New York.

MEI J. y MOSES M.A. (2002): "Art as an Investment and the Underperformance of Masterpieces", American Economic Review, Vol. 92, № 5, pp. 1656-1668.

MEI, J. y MOSES, M.A. (2005): "Vested Interest and Biased Price Estimates: Evidence from an Auction Market", The Journal of Finance. Vol. 60, No5, pp. 2409-2435.

MENDIZÁBAL, A.; MIERA, L.M. y ZUBIA, M. (2002): "El modelo de Markowitz en la gestión de carteras", Cuadernos de Gestión, Vol. 2, No 1, pp. 33-46.

MICHAUD, R.O. (1989): “The Markowitz optimization enigma: Is ‘optimized' optimal?”, Financial Analyst Journal, $\mathrm{N}^{\circ}$ 45, pp.31-42.

PRADO, C.; BLANCO, A. y CONCEJO, A. (2007): El Mercado de los bienes tangibles de colección: especial consideración a la numismática de inversión, SPURJC, Dykinson, Madrid.

PRADO, C. (2009): Factores determinantes en la valoración de los activos numismáticos de oro, Tesis Doctoral, Universidad Rey Juan Carlos, Madrid.

RANDALL, M. R. (1981): "Investment Planning in an Inflationary Environment", Financial Analysts Journal, pp. 68-71.

ROLL, R. (1992): “A mean/variance analysis to tracking error”, The Journal of Portfolio Management, $\mathrm{N}^{\mathrm{o}} 18$, pp. $13-23$.

SALOMON JR., R.S. y LENNOX, M. J. (1984): Financial Assets-A Temporary Setback, Stock Research Investment Policy, Salomon Brothers Inc., United States.

SICONOLFI, M. (1990): "Rare-Coin Dealers Call Summit on Self-Regulation", The Wall Street Journal, 24-08-1990, pp. A7.

SIMAAN, Y. (1997): "Estimation risk in portfolio selection: the mean variance model versus the mean absolute deviation model", Management Science, No 43, pp. 1437-1446.

SHARPE, W. F. (1963): "A simplified Model for Portfolio Analysis", Management Science, Vol. IX, $\mathrm{N}^{\mathrm{o}} 2$, pp. $277-293$.

SHARPE, W. F. (1964): "Capital asset prices: a theory of market equilibrium under conditions of risk", The Journal of Finance, Vol. XIX, Nº 3, pp. 425-442.

SHARPE, W. F. (1966): "Mutual fund performance", Journal of Business, No 39, pp. 119-138.

SHARPE, W.F. y ALEXANDER, G.J. (1990): Investments, Prentice-Hall International Editions, fourth edition, United States, pp. 786-788. (First edition in 1978).

SOLNIK, B. H. (1974): "An Equilibrium Model of the International Capital Market", Journal of Economic Theory, Vol. 8, pp. 500-524. 
Camilo Prado Román / José Luis Coca Pérez / Pablo García Estévez

\section{ANEXO}

Tabla 3

Correlaciones entre los diferentes activos de inversión

\begin{tabular}{|c|c|c|c|}
\hline Numero Columna & Activo & Nuenero Columns & Activo \\
\hline Cotuanna 1 & Oro & Cotumana 11 & MCconm EEUU calided baja \\
\hline Coluzana 2 & M Hea EEUU Calided alta & Coluann 12 & MConm Eq calidad nits \\
\hline Columena 3 & M.Hca EEUU Calided baja & Columana 13 & M. Butlion GB calidad alta \\
\hline Coluenna 4 & MHca GB Calidad baj a & Columna 14 & M Bullion $G B$ calidad baja \\
\hline Coluenna 5 & M Hea Fr Calidad baja & Columna 15 & MC conm Fr calided alta \\
\hline Coluenna 6 & M Hea Em Calidad baj a & Columan 16 & MConm EEUU calided alta \\
\hline Celuzana 7 & M Hea Fr Cenidad alta & Cotumann 17 & Plata \\
\hline Coluzana 8 & M Hea GB Calided eith & Cotumann 18 & Platino \\
\hline Columana 9 & MHca Es Calidad alta & Columann 19 & Peladio \\
\hline Columna 10 & M Bullion EEUU calidad alta & Columann 20 & Rodio \\
\hline
\end{tabular}

\begin{tabular}{|c|c|c|c|c|c|c|c|c|c|c|c|c|c|c|c|c|c|c|c|c|}
\hline & 1 & 2 & 3 & 4 & 5 & 6 & 7 & 8 & 9 & 10 & 11 & 12 & 19 & 14 & 15 & 16 & 17 & is & 19 & $x$ \\
\hline 1 & 1 & & & & & & & & & & & & & & & & & & & \\
\hline 2 & $-0,41$ & t & & & & & & & & & & & & & & & & & & \\
\hline 3 & 0.02 & an & 1 & & & & & & & & & & & & & & & & & \\
\hline 4 & $a v$ & סתג & 0.24 & 1 & & & & & & & & & & & & & & & & \\
\hline 5 & $-0,08$ & 0,33 & 0,25 & 0,35 & 1 & & & & & & & & & & & & & & & \\
\hline 6 & 0.87 & $-0,6$ & 0,11 & 0.85 & 4,24 & 1 & & & & & & & & & & & & & & \\
\hline 7 & ass & azs & D.ss & 0.17 & 0.5s & $a, \theta$ & 1 & & & & & & & & & & & & & \\
\hline 8 & 092 & -0.12 & 0,05 & 0,83 & $-0,40$ & 0,92 & 0.66 & $t$ & & & & & & & & & & & & \\
\hline 9 & 0.91 & $-0,02$ & 0,18 & 0,60 & 4,42 & 0,83 & 0,76 & 0.99 & 1 & & & & & & & & & & & \\
\hline 10 & 0.10 & $=0.10$ & 0.28 & 0.35 & 0.87 & a.3 & -0.58 & c.11 & 0,00 & 1 & & & & & & & & & & \\
\hline 11 & a.s & $-0,06$ & 0.12 & 0,58 & 0,10 & 0,82 & 0,42 & 0.93 & 0.19 & 0.34 & 1 & & & & & & & & & \\
\hline 12 & 0.82 & 0,09 & 0,18 & 0,32 & $-0,59$ & 0,63 & 0.85 & 0.87 & 0.92 & $-0,33$ & 0,77 & 1 & & & & & & & & \\
\hline 13 & $a x 2$ & apt & D.s. & 0.58 & 0,44 & 0,74 & $4.0 s$ & 0,0 & $\Delta, \Delta ?$ & 0.83 & 0.53 & 0.04 & 3 & & & & & & & \\
\hline 14 & Q.2s & a.ss & 0.73 & 0.21 & 4,43 & $a, \infty 0$ & 0.81 & 0.11 & 0.24 & ast & 0.16 & 0,43 & $-0,30$ & 1 & & & & & & \\
\hline 15 & ars & 0,28 & 0,55 & 0,30 & $-0,4 !$ & 0,67 & 0,96 & 0,82 & 0.59 & $-0,32$ & 0,83 & 0,91 & 0,19 & 0,64 & 1 & & & & & \\
\hline Is & 0.48 & Q.48 & 0.07 & 0,28 & 4,13 & 0.61 & 0,44 & 0.82 & 0.82 & $0, n$ & 0,94 & 0,79 & 0,33 & 4,12 & 0,63 & 1 & & & & \\
\hline דג & $a n$ & 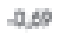 & a.so & 0.35 & 0.34 & 0,74 & س. & 0.00 & D.so & 0,40 & 0.73 & 0.38 & 0.52 & $0.5 \mathrm{t}$ & a.16 & 0.54 & 1 & & & \\
\hline 18 & 0.91 & $-0,59$ & -0.12 & 0,97 & $-0,47$ & 0,93 & 0,39 & 0.82 & 0.76 & $0, n$ & 0,48 & 0,54 & 0,59 & $-0,04$ & 0,51 & 0,42 & 0.84 & 1 & & \\
\hline 19 & 0.83 & 0,53 & 0.37 & 0,83 & 4,34 & 0,83 & 0.21 & o.ss & $a r$ & 0,0 & 0,87 & 0,54 & 0,0 & 4,34 & 0,40 & 0.87 & 097 & 0,50 & 1 & \\
\hline 20 & 0.01 & a.34 & oss & 0,02 & 0,10 & 4,11 & 0,45 & 0.05 & a.03 & 0.28 & 0,34 & 0,03 & 0,13 & 0.72 & 0,37 & 0,41 & 0.31 & 0.03 & 0.35 & 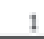 \\
\hline
\end{tabular}


Tabla 4

Correlaciones de los productos utilizados para construir el modelo

\begin{tabular}{|c|c|c|c|c|c|c|c|c|c|}
\hline & Cotuman I & Cohuma 2 & Celluma 3 & Colines 4 & Cotranas 5 & Collunna? & Colimm 10 & Colinna 11 & 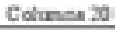 \\
\hline & $\mathrm{Ort}$ & 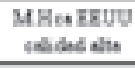 & $\begin{array}{l}\text { MHed EBOU } \\
\text { celidasliege }\end{array}$ & $\begin{array}{l}\text { MHia OB } \\
\text { seldasbes. }\end{array}$ & $\begin{array}{l}\text { MHiafi } \\
\text { caldastest }\end{array}$ & $\begin{array}{c}\text { MHeo Fit } \\
\text { tededes sto }\end{array}$ & 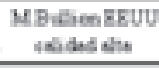 & $\begin{array}{c}\text { MCeen EEUU } \\
\text { asidasbage }\end{array}$ & Rodis \\
\hline Ore & 1 & & & & & & & & \\
\hline $\begin{array}{l}\text { M Nea aruy } \\
\text { cesided sta }\end{array}$ & $-0,4054$ & t & & & & & & & \\
\hline 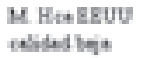 & cosess & 0.7093 & a & & & & & & \\
\hline $\begin{array}{l}\text { M Kes } 9 \mathrm{~B} \\
\text { celided beje }\end{array}$ & Q.350t & $-0,4536$ & 0,215 & $t$ & & & & & \\
\hline $\begin{array}{l}\text { M Heafis } \\
\text { calibes the }\end{array}$ & -0.678 & $0,53 x 7$ & Q.3es & 40.3537 & 1 & & & & \\
\hline $\begin{array}{l}\text { M HiaFi } \\
\text { celded sto } \\
\text { M Ilullion }\end{array}$ & acesos & 0.2913 & $0.5 \pi 72$ & 0.1628 & a,ssas & 1 & & & \\
\hline $\begin{array}{l}\text { EEUV eadidas } \\
\text { she }\end{array}$ & 0,100 & $-0,045$ & $-0,25 \%$ & 0,3605 & 0.6722 & 4,5758 & t & & \\
\hline $\begin{array}{l}\text { M Conn ERUU } \\
\text { caldesthe }\end{array}$ & 0.7325 & 0.0434 & 0.1202 & 0.976 & a.19at & 0,4163 & a.sco & 1 & \\
\hline Reds & 0.0123 & 0.3359 & 0.899 & 0.0293 & 4.1022 & 4.4510 & a.seos & 0.3933 & 1 \\
\hline
\end{tabular}

Composición de las carteras de la Frontera Eficiente. Pesos, rendimientos y riesgos de las 50 carteras de la frontera eficiente. 
Tabla 5

Composición de las carteras de la Frontera Eficiente

\begin{tabular}{|c|c|c|c|c|c|c|c|c|c|c|c|}
\hline & on & 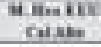 & 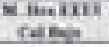 & 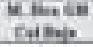 & 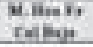 & 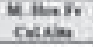 & 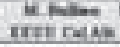 & 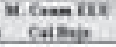 & Nots & $\mathbf{m}$ & Ener \\
\hline 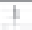 & cas & movis & $\tan 4$ & ins & kns & 130 & ais & 1:av & bis & chs & 130 \\
\hline 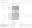 & ens & Jun & 2116 & $97 \pi \mathrm{k}$ & $\operatorname{lnh}$ & toxs & $2 x$ & Ins & 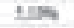 & tits & tas \\
\hline 1 & 120 & throv & the & $4=6$ & in & tost & कas & $\tan$ & $\sin$ & int & kas \\
\hline 4 & enos & nes & : 134 & 1460 & 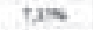 & 1005 & $2 \%$ & ens & T,Mts & 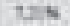 & 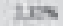 \\
\hline 1 & tast & inest & alat & sians & 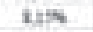 & sast & $2 x$ & $12 x$ & 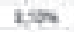 & atis & มี \\
\hline 4 & tass & ints: & Men & Rass & cests & 130 & exs & 1006 & $\sin$ & atas & 230 \\
\hline$t$ & $\operatorname{los}$ & 1306 & $11 x$ & surt & $\cos$ & 130 & $\sec$ & $\cos$ & whe & Nha & 2ues \\
\hline 1 & sas & tas & xas & a an & asirs & $\sin$ & axs & 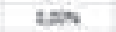 & mais & itsts & uns \\
\hline 1 & exs & ers & nuts & des & $11,3 \mathrm{en}$ & toxs & axe & tans & uses & ins & 26 \\
\hline 13 & $\cos$ & ton & 30 & atios & tars & 120 & 200 & 230 & $143 \mathrm{n}$ & 12186 & Ins \\
\hline t & eas & tas & tas & ans & $12 \pi / 5$ & exs & ans & Mas & Ix) & bess & $14 \pi$ \\
\hline II & exs & texh & $x=0$ & ass & ass & 100 & ses & 1as & wh & Hens & 200 \\
\hline $\mathrm{H}$ & $\operatorname{cas}$ & tast & thes & CU:S & tor & 130 & save & $\tan$ & itm & 400 & Les \\
\hline 14 & expos & tuses & 1445 & ans & $\operatorname{ran}$ & 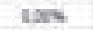 & abes & exos & Inen & 4356 & 24s \\
\hline 13 & exs & tats & wis & itests & tas & 1948 & tase & ant & than & kens & Lats \\
\hline u & enots & nos & ma & sus & Inst & urs & axe & INA & $10, \pi$ & rens & 1 ats \\
\hline 8 & $\cos$ & texs & exp & ness & यis & $\operatorname{sen}$ & $\tan$ & tant & $7,7 \mathrm{~m}$ & ans & 278 \\
\hline ia & $\operatorname{tas}$ & then & ens & HYs & $\operatorname{arn}$ & tast & $0 \times 0$ & $\operatorname{lan}$ & niss & ans & tas \\
\hline is & cast & sust & asts & 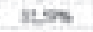 & tex & tast & $\operatorname{axn}$ & $\tan$ & nask & 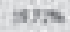 & 15es \\
\hline$=$ & wast & 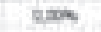 & dins & ine & ans & task & axs & $\tan$ & $7 a t=$ & $x \sin$ & its \\
\hline$=$ & 40. & tes & and & zurs & an & $10 x$ & ans & tas & 142 & nus & 125 \\
\hline 2 & 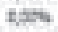 & tan & it:an & ves & $\sin$ & $\tan$ & $2 \times 0$ & $\tan$ & atas & $2 t 20$ & $\operatorname{lin}$ \\
\hline$\Rightarrow$ & ens & tow & nors & xurs & nes & nas & axe & exs & Imans & mins & 10 \\
\hline 3 & $\cos \theta$ & ten & $4,5,5$ & itsen & nesh & sash & $2 x$ & 136 & $\sin$ & thats & ies \\
\hline$y$ & tows & tans & this & iens & ans & $\tan$ & sas & 190 & ans & 3606 & us \\
\hline$x$ & en & sas & ttis & ius & $\tan$ & tars & axes & $\operatorname{lan}$ & Hies & 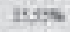 & tes \\
\hline 1 & un & sats & 4.35 & nis & ans & tas & anes & $\tan$ & Hin & 2urs & ars \\
\hline$a$ & 1es & ten & $\pi / s / 4$ & $\operatorname{ten}$ & nas & 1as & 250 & nes & nas & $\pi 25$ & an \\
\hline 3 & 40 & tas & 420 & 200 & ans & 10 & tans & $\tan$ & 11406 & $3: 16$ & in \\
\hline$=$ & 408 & nas & saws & new & ans & Ins & sxo & nas & $143 \mathrm{~m}$ & $7 \mathrm{as}$ & 4re \\
\hline In & ens & texs & Alsth & ness & ans & $\cos$ & $\tan$ & $\tan$ & istors & 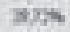 & $48-5$ \\
\hline 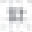 & $\tan$ & tas & eoses & 2005 & ans & $\operatorname{tans}$ & 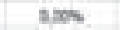 & $\tan$ & atios & Rits & tus \\
\hline$n$ & $\tan$ & tax & tern & Iest & $\operatorname{san}$ & tast & $\operatorname{axs}$ & $\tan$ & thits & ithes & thes \\
\hline 34 & tass & ext & 31350 & 2605 & ans & $\tan$ & 200 & 120 & 4,05 & $22 a 6$ & ists \\
\hline sit & coss & tow & xask & $206 \mathrm{~s}$ & san & tors & $2 x$ & tust & exes & nises & tus \\
\hline 밥 & $t=0$ & tas & ass & ten & an & 120 & ax & $2 \mathrm{ma}$ & $\mathrm{tan}$ & itsts & 10 \\
\hline$w$ & exs & can & 4356 & sas & ans & 120 & thes & UN & 4.6 & $301 \mathrm{~s}$ & inen \\
\hline $\mathbf{z}$ & tars & sus & $\cos$ & 1 15s & nes & ears & 200 & 130 & un & 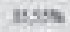 & $11,1=$ \\
\hline$=$ & cass & taps & msts & seos & ans & tas & man & tas & aim & was & Bus \\
\hline 4 & coss & tons & $3.4 \mathrm{~F}$ & nees & non & 1ant & ans & Ins & an & nuss & ives \\
\hline 8 & tas & tath & nso & $2=0$ & ans & 128 & 200 & tan & ats & $a \geq b$ & man \\
\hline$a$ & 2006 & coms & Xis & wows & ans & 100s & pos & tas & nus & ans & Inak \\
\hline 4 & tow & tes & $3 y, 4$ s & 1ets & tims & tans & tures & 120 & uts & $w \%$ & 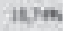 \\
\hline$*$ & $\cos$ & tens & sent & tess & ams & $\operatorname{sen}$ & $2 \times 14$ & axh & $\operatorname{ten}$ & costh & $=1 \mathrm{n}$ \\
\hline 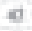 & exs & exs & wors & $\sec$ & airs & tast & exs & tast & $\operatorname{nos}$ & Alats & Rum \\
\hline at & toss & bas & kes & 2006 & tas & 1004 & 200 & 230 & mats & d: & IANh \\
\hline 0 & $\cos$ & wan & uses & yes & $\ln$ & tass & $2 \times 5$ & tacs & neses & arts & 240 \\
\hline a & $\tan$ & tape & ins & 2005 & asen & 100 & bes & 1204 & 12)is & ates & $24 x$ \\
\hline a & cass & thes & Lats & sows & ans & $\tan$ & axs & tast & sens & wess & pusk \\
\hline$=$ & $\tan$ & san & $1=6$ & 1est & $\ln$ & $1=5$ & thes & task & $12 x$ & actist & ates \\
\hline
\end{tabular}


